\title{
Analyse et estimation des puissances de pêche*
}

\author{
Alain Laurec \\ Centre Océanologique de Bretagne, B.P. 337, F-29273 Brest, France
}

\begin{abstract}
- An analysis of the fishing power concepts leads to a distinction between local fishing power (at a given location). and overall fishing power. Overall fishing power takes into account the ability to detect and quickly exploit rich areas.

In order to estimate local fishing power it is necessary to compare the catches of different ships operating in neighbouring areas. Such a comparison performed on two ships will give an estimate of their relative fishing powers. The closer the two ships are, the more reliable will be the estimate. A weighting function must be used, and this function is related to the spatial heterogeneity of the fish density. Such a function is provided by the variogram as defined by Matheron (1965). -

Overall fishing powers can be estimated using Robson's method (1966) where no division into spatial blocks is made: the fact that a ship is in a rich or a poor area must be related to the strategic component of its overall fishing power. A comparison between local and overall fishing powers may give a good insight of the strategic activity of the fishing fleet.

The North East Atlantic albacore (Thunnus alalunga) surface fishery, more precisely its French component, has been studied as an example. The variograms have been estimated and appear to give a significant description of the spatial heterogeneity of the fish density. Individual local and overall fishing powers have been estimated. These calculations give information about the relative influences of local fishing powers and strategic abilities in overall fishing powers.

If the strategic index is defined as the ratio of the overall to the local fishing power it appears that the local fishing power is generally the major component of the overall fishing power of a ship; and that the strategic component cannot be neglected as important differences occur between individual strategic indices. The first result may not be true within the Spanish fleet where some ships are unable to reach the best areas at the beginning of the fishing season.

Some explanations emerge concerning individual local fishing powers and strategic indices. Local fishing power must be related first to type of gear used (bait boats and trollers appear in the fishery), and secondly to the home port of a ship. The strategic index must be essentially related to the home port.

Some statistical inferences are possible only from the estimates of local fishing power. The differences which appear between yearly estimates of the strategic index of the same ships suggest that strong random components affect these estimates.
\end{abstract}

Matheron, G. 1965. Les variables régionalisées et leur estimation. Masson et Cie, Paris. 305 pp. Robson, D. S. 1966. Estimation of the relative fishing power of individual ships. Res. Bull. Inter, Comm. N. W. Atlantic Fish., (3): 5-14

\section{Introduction materiel}

Depuis quelques années est apparu en dynamique des populations le besoin de données d'un type nouveau, à savoir celles issues de carnets de pêche remplis quotidiennement par les pêcheurs eux-mêmes. Ainsi le CNEXO, prenant la suite d'une initiative du Pr Postel soutient un programme de collecte de données par carnets de pêche, ceci pour la pêcherie de surface du germon Nord Atlantique. La procédure de constitution et de gestion informatique des fichiers de données a été decrite par Laurec, Bard, Dao et Branellec (1976). Mais il faut aussi développer des outils théoriques nouveaux, et c'est à la conception d'une nouvelle méthode d'analyse des puissances de pêche qu'est consacrée cette publication. La méthode décrite a été mise au point et testée sur la pêcherie de surface du germon. Les idées qu'elle utilise sont cependant aisément généralisables, à condition de disposer d'une information suffisamment détaillée, c'est-à-dire non seulement du résultat de la pêche, mais encore de la date et du lieu où les captures ont été effectuées.

On notera enfin que les réflexions, remarques ou calculs trop détaillés ont été reportés en annexe afin de ne pas interrompre le fil du discours.

* Contribution n $\mathbf{n}^{\circ} 373$ du Département Scientifique du Centre Océanologique de Bretagne. 


\section{Methode}

\section{Le concept de puissance de pêche}

La nécessité des méthodes d'évaluation des puissances de pêche n'est plus à démontrer en dynamique des populations. La finalité de telles études peut relever de deux points de vue:

soit que l'on cherche à estimer la densité* de poissons à partir des résultats de pêche de navires connus;

soit que l'on cherche à calculer l'effort global sur un stock donné exercé par une flotte composée de navires dont les puissances de pêche individuelles ne sont pas a priori identiques.

La réflexion à ce niveau montre que le concept de puissance de pêche n'est pas aussi bien défini qu'il pourrait y paraître à première vue, et que les deux aspects évoqués ci-dessus peuvent mener à des conceptions différentes. Le rapprochement avec la distinction entre intensité de pêche et effort de pêche, développée par Beverton et Holt (1957), apparaît à cet égard fructueux.

\subsection{Estimation de la densité du poisson: puissance locale}

Dans ce cas, la détermination des puissances de pêche a pour but d'apporter un correctif à l'information brute: le bateau $i$ a pris $N_{i k}$ poissons à tel endroit, le jour $k$, afin de remonter à la densité de poisson à cet endroit à ce jour. Dans cette optique, on ne peut comparer que des navires ayant opéré sur des densités identiques. N'entre donc pas dans la puissance de pêche ainsi estimée l'aptitude à détecter et à se rendre sur des zones plus riches. Pour employer un langage militaire les capacités stratégiques du navire ne sont pas prises en compte.

\subsection{Estimation de l'effort de pêche: puissance globale}

Si le but de l'étude est de quantifier par une mesure de l'effort de pêche la pression à laquelle un stock est soumis, la notion de puissance dite puissance locale développée au paragraphe précédent n'est pas entièrement satisfaisante. La capacité à fréquenter les parties les plus riches de la zone occupée par le stock n'a pas été prise en compte. Ce fait a été

* On parlera constamment de la densité du poisson, supposant implicitement sa capturabilité constante. Si l'on désire éviter cette hypothèse on remplacera la notion de densité par celle du produit: densité $x$ capturabilité. nettement mis en évidence par Poinsard et Le Guen (1975): peut importe en fait de savoir que tel navire disposant d'une grande puissance locale aurait causé une forte mortalité en fréquentant une aire riche s'il n'a pas pu, ou pas su s'y rendre. On ne doit pas mesurer la capacité à prendre plus ou moins de poisson dans une zone de densité donnée, mais bien l'aptitude à prélever une fraction plus ou moins importante d'un stock donné. Pour se convaincre que la distinction introduite n'est pas une pure spéculation de théoricien il suffit de se souvenir que le rayon d'action des divers navires d'une flottille peut parfois grandement varier. Ainsi, dans la pêcherie de surface du germon existe-t-il parmi les navires espagnols de nombreux thoniers de faible tonnage pour qui les parages des Açores, zones les plus riches en juin, sont hors d'atteinte. De même pour les pêcheries multispécifiques certains navires n'exploitent-ils qu'une frange pauvre de l'aire de répartition d'une espèce, car cette zone peut être très intéressante pour une autre espèce.

Si l'on veut s'en tenir à la relation linéaire simple entre mortalité par pêche et effort de pêche, il est fondamental de ne pas sommer les puissances locales mais bien les puissances globales. Il faut tenir compte non seulement de ce que le stock est attaqué plus ou moins massivement, mais aussi de ce qu'il est attaqué dans des conditions de plus ou moins grande vulnérabilité. Pour aller jusqu'au bout de ce raisonnement il faut se poser non seulement le problème de l'aptitude à choisir les lieux de pêche les plus favorables, mais encore à choisir les périodes d'accessibilité maximale du poisson. Un navire n'exploitera pas nécessairement un stock donné durant toute la période où il est accessible.

Le problème est légèrement plus compliqué lorsque l'on prend en compte le temps. Les captures de deux navires ayant pêché en des endroits peut-être différents, à un même moment, donne directement une estimation de leur puissance de pêches globales. Pour comparer deux navires ayant pêché à des instants différents il faudrait prendre en compte les variations d'importance du stock au cours du temps si celles-ci sont sensibles. Pour faire la part des variations de la taille du stock comme de l'accessibilité il faudrait en fait opérer une analyse des cohortes avec une maille temporelle fine.

\subsection{Effort de pêche et intensité de pêche}

Beverton et Holt (1957) distinguent la notion d'effort de pêche, qui est une mesure de la pression à laquelle un stock est soumis, et la notion d'intensité de pêche qui est une mesure de la pression exercée sur une zone géographique donnée. On peut ainsi relier la 
notion d'intensité de pêche à celle de puissance locale, le concept d'effort de pêche à celui de puissance de pêche globale.

Pour surenchérir sur les remarques du paragraphe précédent il est à noter que l'effort de pêche ne saurait être défini comme l'intégrale (au sens mathématique) de l'intensité de pêche, du moins si l'on désire conserver la relation linéaire simple entre effort et mortalité par pêche. Ce problême se pose avec d'autant plus d'acuité que d'une part la densité (ou l'accessibilité) du poisson présente une hétérogénéité spatiale forte, que d'autre part l'intensité de pêche n'évolue pas de la même manière sur toute la zône de pêche. Pour définir un effort de pêche à l'aide des puissances locales on peut utiliser la méthode de Honma (1974).

\section{Les variations spatio-temporelles de la densite du poisson}

Pour pouvoir estimer la puissance locale, il faut parvenir à éliminer l'influence des variations de la densité du poisson. Une première méthode consiste à supposer que ces variations sont régies par une fonction simple.

Ainsi Gulland (1956) ou Robson (1966) supposent l'existence de blocs spatio-temporels de densité uniforme. Ce modèle, qui peut s'avérer trop rigide dans de nombreux cas l'est spécialement pour les grands pélagiques. Stark (1971) suppose que le logarithme de la densité du poisson est un polynome en $t, x$ et $y(t, x$ et $y$ désignant respectivement le temps, la latitude et la longitude). De fait, les fluctuations sont en général trop mouvementées pour qu'un polynome de degré très faible convienne. Or que le degré s'élève et le nombre de coefficients à estimer se multiple et le modèle devient inutilisable en pratique. Le plus raisonnable est alors de supposer simplement une certaine continuité à la densité. Pour préciser ce que l'on entend par continuité, on se référera à la théorie des processus aléatoires, et plus exactement à la théorie des variables aléatoires régionalisées développée par Matheron (1965). La densité du poisson peut alors être décrite par un processus tridimensionnel, les trois dimensions en cause étant le temps, la latitude et la longitude. En un endroit et à un instant donnés, la densité est une variable aléatoire, en un endroit et à un instant proches c'est une seconde variable aléatoire, non indépendante de la précédente. Si $t$ est la date, $x$ la latitude et $y$ la longitude, on notera $d(t, x, y)$ la densité correspondante, $u(t, x, y)$ le logarithme de cette densité: $u(t, x, y)=$ $\log [d(t, x, y)]$. On doit pour obtenir un modèle réaliste supposer que le processus invoqué possède une dérive: $E[u(t, x, y)]=m(t, x, y)$, où $E$ désigne l'espérance mathématique, et $m(t, x, y)$ la dérive évoquée: $u\left(t_{1}, x_{1}, y_{1}\right)-u\left(t_{2}, x_{2}, y_{2}\right)$ est donc une variable aléatoire d'espérance $m\left(t 1, x_{1}, y_{1}\right)-m\left(t_{2}\right.$, $\left.x_{2}, y_{2}\right)$. Pour simplifier dans un premier temps, on supposera que $t_{1}=t_{2}=t . u\left(t, x_{1}, y_{1}\right)-u\left(t, x_{2}\right.$, $\left.y_{2}\right)$ est alors une variable aléatoire d'espérance $m\left(t, x_{1}, y_{1}\right)-m\left(t, x_{2}, y_{2}\right)$.

Une mesure de l'hétérogénéité spatiale est fournie par: $E\left[u\left(t, x_{1}, y_{1}\right)-u\left(t, x_{2}, y_{2}\right)\right]^{2}$.

Lorsque les points $\left(x_{1}, y_{1}\right)$ et $\left(x_{2}, y_{2}\right)$ sont proches géographiquement $m\left(t, x_{1}, y_{1}\right)-m\left(t, x_{2}\right.$, $\left.y_{2}\right)$ est négligeable et $E\left[u\left(t, x_{1}, y_{1}\right)-u\left(t, x_{2}, y_{2}\right)\right]^{2}$ est sensiblement égal à $E\left[u\left(t, x_{1}, y_{1}\right)-m\left(t, x_{1}, y_{1}\right)\right.$ $\left.-u\left(t, x_{2}, y_{2}\right)+m\left(t, x_{2}, y_{2}\right)\right]^{2}$. En supposant que cette quantité ne dépende que de la distance géographique $z_{12}$ des points $\left(x_{1}, y_{1}\right)$ et $\left(x_{2}, y_{2}\right)$ on aboutit donc à une fonction de cette distance $z 12$ qui est une mesure des hétérogénéités spatiales. Cette fonction notée vario $(z)$ constitue ce que Matheron appelle un variogramme. L'hypothèse qui a permis d'y aboutir, que l'on baptisera hypothèse $A$ est discutée en annexe.* Le variogramme vario (z), fonction positive ou nulle croît généralement avec la distance $z$, traduisant en-celà ce que l'on a appelé une certaine continuité. Cependant, il peut ne pas tendre vers zéro avec la distance $z$. On dit alors selon une expression empruntée aux géologues qu'un "effet de pépite" est présent. Cet effet correspond à des phénomènes de microdistribution, que l'on ne cherche pas à élucider en-dessous d'une certaine échelle.

Pour en revenir au problème de départ, si le navire $i$ a pris $N_{i k}$ poissons le jour $k$, en ayant opéré sur une densité $d_{i}$, si de plus la puissance locale de ce navire est $P_{i}$, selon le modèle classique: $\log \left(N_{i k}\right)=$ $\log \left(d_{i k}\right)+\log \left(P_{i}\right)+\varepsilon_{i k}, \varepsilon_{i k}$ étant un résidus inexpliqué.

De même pour le navire $j$ ce même jour:

$$
\log \left(N_{j k}\right)=\log \left(d_{j k}\right)+\log \left(P_{j}\right)+\varepsilon_{j k}
$$

de sorte que:

$$
\begin{aligned}
\log \left(N_{i k} / N_{j k}\right)= & \log \left(P_{i} / P_{j}\right)+\log \left(d_{i k}\right) \\
& -\log \left(d_{j k}\right)+\varepsilon_{i k}-\varepsilon_{j k}
\end{aligned}
$$

$\log \left(N_{i k} / N_{j k}\right)$ apparait ainsi comme la somme de deux termes: le premier $\log \left(P_{i} / P_{j}\right)$ est déterministe, le second $\log \left(d_{i k}\right)-\log \left(d_{j k}\right)+\varepsilon_{i k}-\varepsilon_{j k}$ est alétoire.

La variance de ce terme aléatoire est:

$$
\operatorname{var}\left[\log \left(d_{i k}\right)-\log \left(d_{j k}\right)\right]+\operatorname{var}\left(\varepsilon_{i k}\right)+\operatorname{var}\left(\varepsilon_{j k}\right)
$$

- Les hypothèses, baptisées par des majuscules sont discutées dans l'annexe $B$. 
On ajoute les variances, supposant donc l'indépendance des différents termes. Cette supposition constitue l'hypothèse $B$.

On suppose de plus que $\operatorname{var}\left(\varepsilon_{i k}\right)=\operatorname{var}\left(\varepsilon_{j k}\right)=v$. Cette hypothèse sera dite hypothèse $\mathrm{C}$.

Muni de ce corps d'hypothèses on aboutit ainsi à la formule var $\left[\log \left(N_{i k} / N_{j k}\right)\right]=\operatorname{vario}\left(z_{i j k}\right)+2 v$. Le terme $2 v$ vient donc s'ajouter à l'effet de pépite et l'on notera: $\operatorname{vari}(z)=\operatorname{vario}(z)+2 v$.

Il est alors possible, en disposant de $\log \left(N_{i k} / N_{j k}\right)$ pour différents jours où les navires étaient à la même distance $z$, d'obtenir une estimation de vario $(z)+2 v$, d'après la variance observée sur les $\log \left(N_{i k} / N_{j k}\right)$. L'estimation sera évidemment affinée en moyennant les résultats obtenus pour les différents couples $(i, j)$.

Pour compléter l'étude il faudrait maintenant revenir au cas général, celui où l'on compare la densité en $\left(t_{1}, x_{1}, y_{1}\right)$ et en $\left(t_{2}, x_{2}, y_{2}\right)$, sans que l'on ait nécessairement $t_{1}=t 2$. Le phénomène devient alors plus complexe. On peut ainsi montrer dans l'exemple étudié que si: $x_{1}=x_{2}=x$ et $y_{1}=y_{2}=y$, $E[d(t 1, x, y)-d(t 2, x, y)]$ n'est pas nécessairement négligeable. Le plus sage est donc de ne chercher à comparer que des pêches simultanées. ${ }^{*}$ La programmation s'en trouve, et ce serait en soi un motif suffisant, grandement simplifiée. On fera cependant allusion aux structures temporelles au paragraphe suivant: si les navires $i$ et $j$ ont opéré à une distance inférieure à une distance seuil en deux jours $k 1$ et $k 2$ différents, $\log \left(N_{i k_{1}} / N_{j k_{1}}\right)$ et $\log \left(N_{i k_{2}} / N_{j k_{2}}\right)$ seront supposés stochastiquement indépendants. Cette hypothèse (dite D) repose d'une part sur l'instabilité dans le temps des structures de faible dimension, d'autre part sur la mobilité des navires.

\section{Estimation de la puissance de pêche locale}

\section{1. Puissance de pêche comparée} de deux navires

Lorsque deux bateaux opèrent groupés, la comparaison de leurs pêches donne une idée du rapport de leurs puissances de pêche locales. Même groupés, ils ont probablement rencontré des concentrations différentes. Cependant, moins ils seront proches et plus ces différences auront tendance à être marquées. C'est ce qui est traduit par la fonction vari $(z) d u$ paragraphe II.

Reprenant les notations de ce paragraphe, Log $\left(N_{i k} / N_{j k}\right)$ est une estimation non biaisée de Log $\left(P_{i} / P_{j}\right)$, de variance vari $\left(z_{i j k}\right)$, si $z_{i j k}$ est la distance

* Sur l'exemple étudié, et il en va certainement de même pour beaucoup d'autres, d'un jour sur l'autre la densité et peut-être surtout l'accèssibilité du poisson, varient notablement. des navires $i$ et $j$ le jour $k$. Si l'on ne retient que les jours où les deux navires étaient distants de moins de $z c, z c$ étant suffisamment faible, les estimations $\log \left(N_{i k} / N_{j k}\right)$ correspondant à des jours différents peuvent être considérées comme statistiquement indépendantes.

On dispose ainsi d'une série d'estimations indépendantes de $\log \left(\boldsymbol{P}_{\boldsymbol{i}} / \boldsymbol{P}_{j}\right)$. Une moyenne pondérée par les variances fournira donc l'estimation globale, notée $P_{i j}$, de $\log \left(P_{i} / P_{j}\right)$.

$$
P_{i j}=\frac{1}{M_{i j}} \sum_{k} w_{i j k} \log \left(N_{i k} / N_{j k}\right)
$$

Si $M_{i j}=\sum_{k} w_{i j k}$

$$
\begin{aligned}
& \text { avec } \quad w_{i j k}=\frac{\operatorname{vari}(0)}{\operatorname{vari}\left(z_{i j k}\right)} \quad \text { si } z_{i j k}<z c \\
& w_{i j k}=0 \quad \text { si } z_{i j k}>z c
\end{aligned}
$$

Parmí les combinaisons linéaires des $\log \left(N_{i k} / N_{j k}\right)$ telles que $w_{i j k}$ soit nul si $z_{i j k}>z c$, cet estimateur est l'estimateur du maximum de vraisemblance. La notion de distance maximale a un double but: assurer que c'est bien la puissance locale qui est estimée (cf. § 1-4); garantir l'indépendance des divers estimateurs journaliers.

\section{2. Puissances de pêche par rapport à un ensemble de référence}

III. 2.1. L'ensemble de référence. A l'issue du paragraphe III 1. on dispose d'une matrice de $P_{i j}$ qui sont les estimations des $\log \left(P_{i} / P_{j}\right)$. Il est évident que de toute façon les $\log \left(P_{i}\right)$ calculés d'après ces $P_{i j}$ seraient définis à une constante additive près, puisque seules importent les quantités $\log \left(P_{i}\right)-$ $\log \left(P_{j}\right)$. Une condition supplémentaire est donc nécessaire pour parvenir à un système unique de $\log \left(P_{i}\right)$. La solution la plus simple consiste à choisir un navire io comme standard, et à fixer ainsi arbitrairement sa puissance à 1 . Cette procédure comporte un danger, car comme tous les résultats de pêche ceux du navire de référence comportent une part aléatoire, composante qui va affecter l'estimation de toutes les autres puissances. Il est par conséquent plus prudent d'imposer une condition du type: $\sum_{i} u_{i} . \log \left(P_{i}\right)=0$.

Les $u_{i}$ sont des nombres positifs ou nuls, l'ensemble des navires $i$ tels que $u_{i}$ soit non nul constitue l'ensemble de référence. Ce peut être la flottille au complet si par exemple $u_{i}=1$ pour tout $i$. Ce sont alors les variations des $\log \left(P_{i}\right)$ par rapport à leur moyenne qui sont étudiées.

Le danger évoqué précédemment n'est pas sup- 
primé. Il est cependant grandement réduit: une moyenne est moins soumise aux fluctuations aléatoires que chacun des termes qui la composent.

\section{2.2. Une méthode d'estimation des puissances de} pêche individuelles. On notera désormais $l p_{i}=\log$ $\left(P_{\xi}\right)$. On dispose donc à ce niveau de l'étude d'une matrice de $\boldsymbol{P}_{i j}$, estimations de $l p_{i}-l p_{j}$. De plus une condition linéaire supplémentaire a été choisie. Le calcul montre aisément que la variance de $p_{i j}$ est vari(0) $\times 1 / M_{i j}$ avec comme il a été dit $M_{i j}=\sum_{k} w_{i j k}$.

L'idée la plus simple consiste donc à minimiser la quantité:

$$
\sum_{i, j} M_{i j}\left[\left(l p_{i}-l p_{j}\right)-p_{i j}\right]^{2}
$$

En développant les calculs on aboutit au système: $\sum_{j} c l_{i j} l_{j}=a l_{i} i=1, N b$ si $N b$ est le nombre de navires étudiés, avec $c l_{i j}=M_{i j}$ pour $i \neq j$

et

$$
c l_{i i}=-\sum_{j \neq t} M_{i j}
$$

$$
a l_{i}=\sum_{j \neq t} M_{i j} \times p_{i j} .
$$

S'ajoute à ce système linéaire qui, tel quel comporte une infinité de solutions, la condition: $\sum_{i} u_{i} l p_{i}=0$.

Une des équations du système de départ peut être supprimée et remplacée par la condition supplémentaire. Le système final s'écrit donc:

$$
\sum_{j} c_{i j} \times l_{j}=a_{i} \text { avec } c_{i j}=c l_{i j} \text { sauf } i=i \text { io quelconque, }
$$
et $c_{i 0 j}=u_{j}$, ainsi que $a_{i}=a l_{i}$ sauf $a_{i 0}=0$.

Ce système de $N b$ équations à $N b$ inconnues est résoluble en général, et si l'on note $b_{i j}$ les termes de la matrice inverse de la matrice des $c_{i j}$ la solution sera fournie par $l_{p}=\sum_{j} b_{i j} \times a_{j}$.

L'estimation des $\log \left(p_{i}\right)$ ainsi obtenue n'est pas l'estimation du maximum de vraisemblance. Pour obtenir celle-ci, il faudrait disposer non seulement de la variance des $p_{i j}$, mais de leur matrice de variance-covariance complète. La taille de cette matrice est cependant telle qu'il est sans espoir de chercher à l'utiliser dans un programme sur ordinateur, programme qui est nécessairement l'aboutissement d'une méthode de calcul.

\section{3. Variance-covariance des estimations obtenues}

Le système final du paragraphe III 2.2. peut s'écrire matriciellement (les majuscules correspondent aux matrices et par exemple $A$ est la matrice des $a_{i j}$ ). $C \times L P=A$ d'ou $L P=B \times A$ avec $B=C^{-1}$.
La matrice de variance-covariance de $L P$ est alors égale à: ${ }^{t} B \times \operatorname{COVA} \times B$, où ${ }^{t} B$ désigne la transposée de $B$, COVA la matrice de variance-covariance du vecteur $\mathrm{A}$.

Il faut donc disposer de cette matrice COVA. Comme $a_{i}=a l_{i}$ pour tout $i$ sauf pour $i=i 0$ car $a_{i 0}=0$, le problème est résolu si la matrice de variance-covariance des $a l_{i}$ est connue.

$$
a l_{i}=\sum_{j \neq i, k} w_{i j k} \log \left(N_{i k} / N_{j k}\right) .
$$

Du fait de l'hypothèse d'indépendance des Log $\left(N_{i k} / N_{j k}\right)$ pour différents $k$, lorsque les comparaisons correspondant à des distances trop grandes sont éliminées, la covariance entre $a l_{i 1}$ et $a l_{i 2}$ est donnée par:

$$
\begin{aligned}
k_{, j 1} \neq i 1, j 2 \neq 12 & w_{i 1 j 1 k} w_{i 2 j 2 k} \\
& \operatorname{cov}\left[\log \left(N_{i 1 k} / N_{j 1 k}\right), \log \left(N_{i 2 k} / N_{j 2 k}\right)\right]
\end{aligned}
$$

Il suffit donc de connaître les covariances

$$
\operatorname{cov}\left[\log \left(N_{i 1 k} / N_{j 1 k}\right), \log \left(N_{i 2 k} / \mathbf{N}_{j 2 k}\right)\right] \text {. }
$$

Le calcul de ces valeurs, quelque peu douloureux a été reporté dans l'annexe $\mathrm{C}$.

\section{4. Inférences statistiques}

Il apparaît légitime de supposer le vecteur $\mathrm{A}$ distribué de façon multinormale. En effet, il est formé par la somme de contributions journalières, contributions stochastiquement indépendantes: on est donc en droit d'invoquer le théorème central limite multivariable démontré entre autres par Anderson (1958).

Supposer que A est distribué de façon multinormale équivaut à supposer que $L P$ l'est. Sous cette hypothèse, il devient possible de bâtir des intervalles de confiance pour les $\log \left(P_{i}\right)$ et partant pour les $P_{i}$. Il est cependant bien connu qu'une estimation non biaisée des $\log \left(P_{i}\right)$ ne conduit pas à une estimation non biaisée des $P_{i}$. Il est alors possible d'appliquer la correction de Laurent (1963). Mais il importe de garder à l'esprit que si l'on travaille essentiellement sur les logarithmes, tant des captures que des puissances, le plus sage est de conserver l'estimateur non biaisé des $\log \left(P_{i}\right)$ [car en retour à un estimateur non biaisé des $\boldsymbol{P}_{\boldsymbol{i}}$ correspond un estimateur biaisé des $\left.\log \left(P_{i}\right)\right]$.

Enfin l'hypothèse de multinormalité, et l'estimation de la matrice de variance-covariance permettent le test d'hypothèses linéaires: on peut tester que la moyenne, au besoin pondérée, des logarithmes des puissances des navires d'une certaine catégorie est ou non supérieure à la moyenne des puissances dans un autre groupe. On peut de même calculer la variance 
d'une telle moyenne et en déduire des intervalles de confiance.

\section{Estimation de la puissance de pêche globale}

Dans le paragraphe II il a été considéré que lorsque deux navires opéraient groupés, le fait qu'ils aient rencontré des densités plus ou moins grandes de poisson, relevait du simple hasard. Si cela est vrai quelle que soit la distance des deux navires, il n'y a pas lieu de faire la distinction entre puissance de pêche in situ et puissance de pêche globale. Il ne peut y avoir de capacités stratégiques car nulle stratégie n'est possible. S'il y a vraiment des zones, plus riches et si certains navires ont plus que d'autres la capacité de les détecter et de s'y rendre, la distinction s'impose.

On a vu dans le paragraphe précédent comment estimer la puissance de pêche locale. Pour estimer la puissance de pêche globale le plus simple est alors d'utiliser la méthode de régression de Robson (1966). Cependant, on ne procèdera pas à un découpage par blocs spatiaux où la densité serait supposée uniforme. Il y a un seul bloc spatial recouvrant l'ensemble de la zone de pêche, où la densité n'est surement pas uniforme. Mais le fait qu'un navire ait ou non pu se trouver sur des zones riches fait partie de sa puissance de pêche globale. Disposer de la puissance de pêche globale et de la puissance locale permet même d'extraire de celle-là la composante "capacité stratégique". Pour en revenir à la méthode de Robson la seule division restante sera la cassure en blocs temporels.* Les aspects statistiques de la méthode ne sont cependant plus intéressants: les résidus d'un jour sur l'autre ne sont certainement pas indépendants (ils ne peuvent l'être dès que les blocs spatiaux utilisés sont larges). Reste donc un simple ajustement par les moindres carrés.

\section{Resultats}

\section{Programmation}

Une méthode faisant appel à des calculs lourds n'a d'utilité que si elle est programmable. La méthode de Robson (1966) dont une variante simplifiée a été préconisée pour calculer les puissances globales, a depuis longtemps été programmée, c'est un program-

- La différence entre puissances de pêche globale et in situ relève de la capacité à choisir les zones intéressantes et à s'y rendre. De même, la différence entre puissance de pêche globale estimée comme on l'indique et celle que l'on pourrait obtenir sans découpage en blocs temporels, comparant donc simplement les rendements moyens sur une saison de pêche, relèverait de la capacité à choisir les périodes les plus intéressantes pour la pêche. me dô à C.L. Berude, décrit dans le rapport technique FAO $\mathrm{n}^{\circ} 101$ (Abramson, 1971).

La méthode exposée de calcul des puissances locales a été programmée par nos soins. En fait, deux programmes sont nécessaires, le premier détermine l'allure du variogramme ou plus exactement de la fonction notée vari $(z)$. Le second programme effectue le calcul des puissances de pêche. Une programmation extrêmement soignée est nécessaire si l'on désire avoir accès aux variances-covariances sans sombrer dans des temps de calcul prohibitifs. Sans entrer dans une description des programmes écrits, un ordre de grandeur du temps et de la place requis peut être utile: pour 50 bateaux et une saison de 100 jours de pêche la place requise est de $20 \mathrm{k}$ mots, le temps nécessaire de 1 minute 30 , ceci pour le second programme, le premier occupant une place inférieure et nécessitant un temps de calcul beaucoup plus faible. Place mémoire et temps requis s'entendent sur un ordinateur CII 10070.

\section{Application à la pêcherie de surface du germon (Thunnus alalunga) Nord Atlantique}

C'est plus exactement la flottille française qui va être étudiée, car nous ne disposons pas de carnets remplis par les pêcheurs espagnols. Une étude détaillée sera publiée ultérieurement et seront simplement présentés ici quelques résultats à titre d'illustration. L'année 1972 sera plus spécialement étudiée, car c'est une année charnière dans l'histoire de la pêcherie. Les captures globales en nombre sont seules étudiées, une étude par classe d'âge étant destinée à la publication ultérieure annoncée précédemment.*

\section{1. Allure du variogramme et de la fonction vari (z)}

Variogramme et fonction vari $(z)$ ne différent que d'une constante. On peut ainsi analyser l'allure du variogramme à l'aide de la fonction vari $(z)$.

Les études ont été faites sur huit années (1967 à 1974). Les fonctions vari $(z)$ ont été estimées séparément pour le début et la fin de chaque année d'une part, pour l'Ouest et l'Est de la zone de pêche d'autre part. Des variations apparaissent certes, mais pour chaque année une fonction vari $(z)$ unique peut être utilisée sans erreur grave. Lorsque les distances n'excèdent pas quelques dizaines de miles cette courbe peut être assimilée à une droite, ou pour obtenir

* Quant apparaissent des variations d'accessibilité avec l'age, variations susceptibles de différer d'un engin voire d'un bateau à l'autre la rigueur conduit à calculer des puissances pour une classe d'age donnée. 


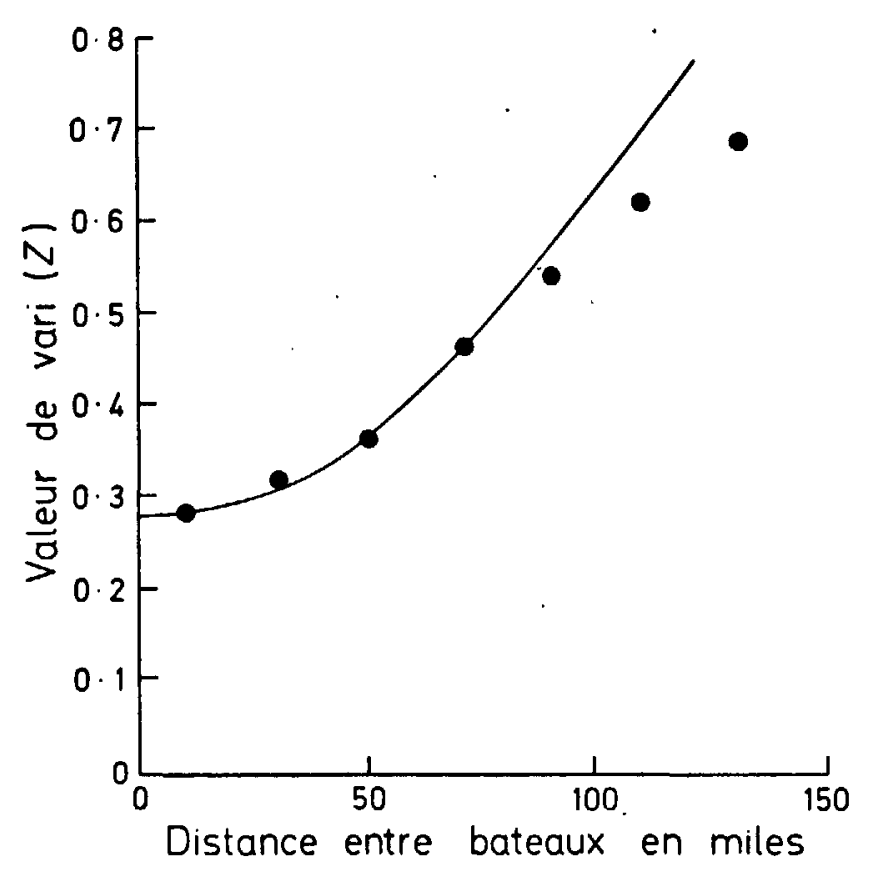

Figure 1. Estimation de la fonction vari (z).

une meilleure adéquation à un fragment de parabole à tangeante horizontale à l'origine.

La Figure 1 montre pour l'année 1972 les points estimés et l'ajustement (à l'oeil) de la parabole. Cet ajustement ne vaut qu'en deçà d'une soixantaine de miles. De toutes façons pour des distances critiques supérieures à soixante miles l'indépendance stochastique d'un jour sur l'autre des comparaisons n'est plus assurée.

L'allure du variogramme peut être utilisée pour décrire les hétérogénéités spatiales de la densité du poisson. Ainsi il apparaît qu'en des points distants de vingt ou trente miles, il n'apparaît pas d'écart sensible de densité. En revanche en étudiant le variogramme pour des distances importantes on verrait celui-ci tendre vers un palier, montrant qu'au-delà d'une distance, que l'on appelle la portée, apparaît une certaine indépendance des densités respectives. D'une année sur l'autre l'allure générale des variogrammes reste constante. La portée peut cependant changer, de même que la valeur de vari(0). On peut se demander si ces seconds changements ne sont pas das à une amélioration du report des données sur les carnets de pêche car vari(0) décroît régulièrement d'année en année.

La valeur à l'origine de vari(z), soit en 1972 sensiblement 0.28 montre quelle confiance on peut accorder à la capture en un jour donné d'un navire isolé comme indice de la densité de la zone fréquentée. On peut exprimer la densité logarithmique par l'espérance du logarithme des captures d'un navire de puissance locale 1 , notée $\log (d)$. Le logarithme de la pêche sur un jour d'un navire de puis- sance locale 1 sera alors une variable aléatoire d'espérance $\log (d)$ et de variance $0 \cdot 14$ (soit $0 \cdot 28 / 2$ ). Si la capture indiquée est de 100 thons, l'intervalle de confiance à $5 \%$ sera $(48,208)$. Le calcul peut être compliqué au cas de plusieurs navires. On voit le parti que l'on peut tirer de telles considérations pour définir une stratégie de conduite des flottilles.

\section{2. Estimation des puissances locales}

La distance critique utilisée est de quarante miles. De fait dès l'instant où l'on utilise une distance critique supérieure à trente miles les résultats sont stables vis-à-vis de changements de la distance critique.

Dans la pêcherie deux types de pêche sont pratiqués: la pêche à la ligne traînante (ligneurs) et la pêche à l'appât vivant (canneurs). L'ensemble de référence est constitué par les ligneurs:

$$
\begin{aligned}
& \sum_{i} u_{i} \log \left(p_{i}\right)=0 \\
& u_{i}=0 \text { pour un canneur } \\
& u_{i}=1 \text { pour un ligneur }
\end{aligned}
$$

en reprenant les notations déjà utilisées.

Pour l'année 1972, quatre canneurs ont rempli des carnets de pêche. Leur puissance locale respective est $1 \cdot 06,1 \cdot 76,0.89,1 \cdot 29$. La précision de ces estimations est relativement médiocre car les canneurs n'opèrent pas très souvent au voisinage des ligneurs. La moyenne géométrique des puissances locales des canneurs est $1 \cdot 22$. L'intervalle de confiance à $5 \%$ de cette moyenne est 1.05, 1.39. A l'imprécision sur cette moyenne s'ajoute l'incertitude quant à l'écart entre elle et la moyenne sur l'ensemble des canneurs. Cumulée sur plusieurs années, l'étude permet cependant de conclure à la plus grande puissance locale des canneurs. Sans entrer dans les détails la valeur la plus raisonnable pour la puissance locale du canneur typique est de $1 \cdot 30$.

A l'intérieur de la classe des ligneurs les puissances individuelles vont de 0.73 à 1.56 , avec des intervalles de confiance à $5 \%$ respectivement $(0.64,0.84)$ et $(1.34,1.83)$. Entre 0.9 et 1.1 se regroupent $30 \%$ des puissances locales individuelles. Il est très difficile de relier ces puissances aux caractéristiques matérielles du thonier. Ligne d'eau, bruit et sillage sont probablement des éléments déterminants mais difficillement quantifiables ou inconnus dans l'immédiat. Un facteur prépondérant apparaît cependant, c'est le port d'attache du navire. La troisième colonne du Tableau 1 regroupe les puissances locales des thoniers de quatre ports notés $A, B, C$ et $D$ pour ne pas envenimer les querelles locales. L'écart systématique 
Tableau 1. Logarithme des puissances globales, locales et des capacités stratégiques individuelles

\begin{tabular}{|c|c|c|c|}
\hline Port & $\log \left(P g_{i}\right)$ & $\log \left(P l_{i}\right)$ & $\log \left(C s_{i}\right)$ \\
\hline A & 0.21 & 0.17 & 0.04 \\
\hline A & 0.05 & 0.22 & -0.17 \\
\hline A & 0.41 & 0.45 & -0.04 \\
\hline A & 0.32 & $0 \cdot 30$ & 0.02 \\
\hline A & -0.03 & -0.06 & 0.03 \\
\hline B & 0.08 & 0.06 & 0.02 \\
\hline B & -0.06 & -0.20 & $0 \cdot 14$ \\
\hline B & $0 \cdot 19$ & 0.01 & $0 \cdot 18$ \\
\hline B & -0.07 & -0.20 & $0 \cdot 13$ \\
\hline B & $0 \cdot 16$ & $0 \cdot 14$ & 0.02 \\
\hline B & $0 \cdot 19$ & $0 \cdot 10$ & 0.09 \\
\hline B & 0.03 & -0.07 & $0 \cdot 10$ \\
\hline B & $0 \cdot 15$ & 0.24 & -0.09 \\
\hline B & 0.23 & 0.25 & -0.02 \\
\hline B & -0.09 & -0.06 & -0.03 \\
\hline B & -0.04 & -0.04 & 0.00 \\
\hline C & 0.02 & -0.08 & $0 \cdot 10$ \\
\hline C & -0.09 & 0.00 & -0.09 \\
\hline C & 0.04 & $0 \cdot 10$ & -0.06 \\
\hline C & -0.07 & 0.00 & -0.07 \\
\hline C & $-0 \cdot 15$ & -0.07 & -0.08 \\
\hline C & 0.08 & $0 \cdot 19$ & -0.11 \\
\hline C & -0.09 & 0.03 & -0.06 \\
\hline C & 0.01 & $0 \cdot 17$ & $-0 \cdot 16$ \\
\hline D & -0.27 & -0.34 & 0.07 \\
\hline D & -0.25 & $-0 \cdot 14$ & $-0 \cdot 11$ \\
\hline D & 0.00 & $0 \cdot 16$ & $-0 \cdot 16$ \\
\hline D & -0.01 & -0.21 & $0 \cdot 20$ \\
\hline D & -0.05 & $-0 \cdot 16$ & $0 \cdot 11$ \\
\hline D & -0.32 & -0.28 & -0.04 \\
\hline D & 0.01 & -0.23 & $0 \cdot 24$ \\
\hline D & 0.09 & $0 \cdot 17$ & -0.08 \\
\hline D & -0.22 & -0.28 & 0.06 \\
\hline D & -0.12 & -0.23 & $0 \cdot 11$ \\
\hline D & -0.06 & -0.03 & -0.03 \\
\hline D & -0.21 & -0.21 & 0.00 \\
\hline D & -0.02 & -0.21 & 0.19 \\
\hline D & -0.09 & -0.04 & -0.05 \\
\hline D & 0.00 & -0.16 & $0 \cdot 16$ \\
\hline
\end{tabular}

qui semble apparaître peut être confirmé statistiquement. Là encore le problème d'inférence apparaît à deux niveaux: inférence des estimations aux valeurs réelles des puissances locales individuelles, inférence de l'échantillon des thoniers ayant rempli des carnets de pêche à la flottille complète. Le problème a priori provient de ce que les estimations individuelles sont stochastiquement liées. Cependant, leur lien est assez faible comme le montrent des coefficients de corrélation très bas. On le négligera donc pour se replacer dans le cadre statistique classique où les estimations des puissances locales individuelles sont autant de réalisations indépendantes d'une variable aléatoire (intégrant les deux niveaux évoqués plus haut).

Un test $t$ sur les puissances logarithmiques montre que le port $\mathrm{D}$ présente une puissance locale moyenne plus faible que les autres ports, avec un seuil de significativité à $1 \%$.

D'une année sur l'autre les puissances locales individuelles sont relativement stables. On peut améliorer la précision en effectuant une étude cumulée sur plusieurs années. D'une année sur l'autre quelques variations notables peuvent apparaître (beaucoup plus marquées dans l'ensemble que celles intervenant entre début et fin d'année). Ceci est probablement da à des modifications techniques ou à des changements dans l'équipage.

\section{3. Estimation des puissances globales. Comparaison des deux types de puissance}

Pour mieux comparer les résultats obtenus à ceux fournis par l'estimation des puissances locales, la référence à l'ensemble des ligneurs a été conservée. Par différence entre le logarithme de la puissance globale et celui de la puissance locale, on obtient ce que l'ont peut définir comme le logarithme de l'indice stratégique. Si l'on note respectivement $P g_{i}, P l_{i}$ et $C s_{i}$ la puissance globale, la puissance locale et l'indice stratégique du bateau $i: P g_{i}=P l_{i} \times C s_{i}$ soit $\log \left(P g_{i}\right)=\log \left(P l_{i}\right)+\log \left(C s_{i}\right)$. La moyenne des $\log \left(C s_{i}\right)$ sur l'ensemble des thoniers ligneurs sera nulle de façon évidente.

Il n'apparaît pas d'écart systématique convaincant des capacités stratégiques des ligneurs et des canneurs. Ceci dit là encore il faut souligner la faiblesse de notre échantillon, faiblesse accusée par la dispersion des puissances et capacités stratégiques individuelles.

A l'intérieur des ligneurs, les puissances globales vont de 0.73 à 1.51 , avec un peu moins de la moitié des puissances entre 0.9 et $1 \cdot 10$. Les indices de capacité stratégique vont de 0.79 à $1.24,60 \%$ étant comprises entre 0.9 et $1 \cdot 10$.

Il est à noter que la dispersion est plus forte pour les puissances locales que pour les puissances globales. La prise en compte des capacités stratégiques homogénéise les puissances.

Les variations de l'indice stratégique s'éclairent là encore si l'on se réfère au port d'attache (les flottilles de chaque port ont d'ailleurs tendance à se grouper). Dans le Tableau 1 ont été reportés pour les quatre ports choisis le logarithme des puissances individuelles globales et locales et le logarithme des indices stratégiques. Le Tableau 2 présente les moyennes pour chaque paramètre et chaque port. Le port $D$ montre des puissances locales médiocres mais de bonnes capacités stratégiques. Il est à noter que dans cette pêcherie qui est souvent à la limite de la rentabilité, tout au moins pour les pêcheurs français, un thonier cumulant une mauvaise puissance locale et 
Tableau 2. Moyennes port par port des logarithmes des puissances globales et locales ainsi que des capacités stratégiques

\begin{tabular}{cccc}
\hline Port & $\begin{array}{c}\text { Moyenne des } \\
\log \left(P g_{i}\right)\end{array}$ & $\begin{array}{c}\text { Moyenne des } \\
\log \left(P l_{i}\right)\end{array}$ & $\begin{array}{c}\text { Moyenne des } \\
\log \left(C s_{i}\right)\end{array}$ \\
\hline A & 0.20 & 0.22 & -0.02 \\
B & 0.07 & 0.02 & 0.05 \\
C & -0.03 & -0.04 & -0.07 \\
D & -0.10 & 40.15 & 0.05 \\
\hline
\end{tabular}

une capacité stratégique médiocre ne pourrait persévérer.

On pourrait être tenté d'étayer les raisonnements par des tests statistiques du type test $t$. Des problèmes apparaissent comme pour les puissances locales car les estimations des puissances globales individuelles sont corrélées entre elles, et cette fois de façon que l'on ne sait pas chiffrer.

Il est à noter que d'une année sur l'autre les indices stratégiques se conservent moins bien que les puissances locales. Les fluctuations ne sont cependant pas erratiques, auquel cas ceci signifierait que les capacités stratégiques seraient égales entre thoniers, et que seul le hasard ferait attribuer sur une année un indice stratégique plus ou moins grand à un navire. En effet par exemple le port $C$ présente chaque année un indice stratégique logarithmique moyen négatif.

Pour préciser l'influence relative de la puissance locale et de l'indice stratégique on peut s'en référer à la moyenne en valeur absolue, ou à la moyenne quadratique des $\log \left(P l_{i}\right)$ et des $\log \left(C s_{i}\right)$. Dans les deux cas la puissance locale apparaît comme la composante essentielle, sans que l'on puisse négliger l'indice stratégique.

\section{Discussion}

La première conclusion a trait à l'utilité du formalisme des variables aléatoires régionalisées. La notion de variogramme est certainement un concept utile, et pourrait notamment être appliquée à des problèmes d'échantillonnage.

La distinction des deux concepts de puissances de pêche apparaît comme recouvrant bien une réalité concrète sur l'exemple étudié. Cette distinction permet de calculer au mieux les puissances locales nécessaires à l'établissement d'une cartographie des c.p.u.c. Elle permet d'autre part de mieux comprendre et d'expliquer la puissance globale en la séparant en deux composantes: capacité stratégique et puissance locale.
$\mathrm{Ce}$ fait est probablement utile dans toutes les pêcheries où l'activité de localisation des concentrations et de déplacement sur ces concentrations est importante.

La méthode proposée de calcul des puissances locales s'avère quant à elle opérationnelle. Lorsque la distinction entre les deux puissances ne s'impose pas, elle peut encore fournir une méthode d'évaluation des puissances présentant deux avantages: pondération des diverses comparaisons des captures de deux navires, cette pondération jouant en faveur des instants où deux navires sont proches; inférence statistique reposant sur une hypothèse moins naîve et brutale que celle des méthodes usuelles.

\section{Remerciements}

Il m'est un devoir et un plaisir de remercier les pêcheurs qui ont bien voulu remplir des carnets de pêche, ainsi que Messieurs F. X. Bard et J. C. Dao qui ont mené le programme de distribution et de collecte de ces carnets, avant de m'éclairer de leur grande connaissance de la pêcherie dans l'étude des résultats.

\section{References}

Abrahamson, N. J. 1971. Computer programms for Fish Stock Assessment. F.A.O. Fish. Biol. tech. Pap., 101: 4 pp. + unpag.

Anderson, T. W. 1958. An introduction to multivatiate statistical analysis. Wiley \& Sons. N.Y., 374 pp. See p. 74-9.

Beverton, R. et Holt, S. 1957. On the dynamics of exploited fish populations. Fishery Invest., Lond., Ser. 2, 19: 533 pp.

Gulland, J. A. 1956. On the fishing effort in English demersal fisheries. Fishery Invest., Lond., Ser. 2, 20: (5), 41 pp.

Honma, M. 1974. Estimation of effective overall fishing intensity of tuna longline fishery - Yellowfin tuna in the Atlantic Ocean as an example of seasonally fluctuating stocks. Bull. Far Seas Fish. Res. Lab., (10), 63-75.

Laurec, A., Bard, F. X., Dao, J. C. et Branellec, J. 1976. Sous presse. La gestion informatique des données relatives à la pêcherie de surface du germon dans le nord-est Atlantique. Rapp. Sci. Tech. CNEXO. $45 \mathrm{pp}$.

Laurent, A. G. 1963. Lognormal distribution and the translation method: description and estimation problems. J. Amer. statist. Ass., 58: 231-5.

Matheron, G. 1965. Les variables régionalisées et leur estimation. Masson et Cie. Paris. 305 pp.

Poinsard, F. et Le Guen, J. C. 1975. Observations sur la définition d'une unité d'effort de pêche applicable à la pêcherie de thon de l'Atlantique Tropical Africain. Rapp. R.-v. Réun. Cons. int. Explor. Mer, 168: 39-43. 
Robson, D. S. 1966. Estimation of the relative fishing power of individual ships. Res. Bull. Int. Commn. NW Atlant. Fish, (3): 5-14.
Stark, A. E. 1971. A computer programm to estimate fishing power by the method of fitting constants. J. Cons. int. Explor. Mer, 33: 478-82.

\section{Annexe A}

\section{Méthode simplifiée de calcul des puissances locales}

La distance de deux navires a été considérée comme une donnée. On peut cependant envisager que seul le fait que la distance de deux bateaux soit inférieure ou non à une distance seuil $z c$ soit connu, les distances inférieures à $z c$ étant distribuées de façon aléatoire.

Ainsi $\log \left(N_{i k} / N_{j k}\right)=\log \left(P i / P_{j}\right)+\varepsilon_{i j k}$, lorsque les bateaux $i$ et $j$ sont à une distance inférieure à $z c$. La variance de $\varepsilon_{i j k}$, supposée indépendante de $k$ est estimée d'après la dispersion des différentes valeurs observées des $\log \left(N_{i k} / N_{j k}\right)$. Supposant que cette variance ne dépend pas de $i$ et $j$ on améliorera son estimation en moyennant les estimations obtenues pour les différents couples $(i, j)$. On peut de même que pour la méthode complète présentée dans le paragraphe III supposer et vérifier que lorsque $z c$ est faible les $\varepsilon_{i j k}$ sont statisquement indépendants pour les différentes valeurs de $k$. Les calculs se simplifient alors légèrement (d'ou l'expression méthode simplifiée). En contrepartie, une certaine information est négligée puisque l'on considère que les distances inférieures à $z c$ sont distribuées de façon aléatoire alors que l'on pourrait connaître leur valeur exacte.

La différence de point de vue des deux méthodes, celle dite complète et celle dite simplifiée mérite que l'on s'y attarde. Pour parfaire la formalisation mathématique il faudrait en fait considérer que la position des différents navires correspond à autant de variables aléatoires liées entre elles, et liées à la densité du poisson, densité elle-même aléatoire. Lorsque l'on suppose connu l'emplacement des divers navires, ou le fait que ces navires soient ou non distants de plus de $z c$ on raisonne en termes de probabilités conditionnelles. Les divers points de vue correspondent à des probabilités conditionnées par différents événements.

En pratique, lorsque la puissance locale est mal individualisée, les capacités stratégiques jouant très vite dès que $z c$ croit, la puissance estimée change de nature lorsque $z c$ varie. Il peut alors être utile d'appliquer la méthode simplifiée pour diverses valeurs de $z c$. La méthode dite complète risque de masquer le problème par le poids fort attribué aux comparaisons effectuées les jours où les navires sont très proches.

\section{Annexe B}

\section{Notes sur les principales hypothèses evoquées}

Toute méthode de calcul suppose une formalisation mathématique et donc un certain nombre d'hypothèses, qui ne sont jamais qu'imparfaitement satisfaites. Il est hors de propos de faire un examen exhaustif des possibilités d'écart à ces hypothèses. Il est cependant nécessaire de réfléchir sur la signification réelle des postulats invoqués, de s'assurer de leur réalisme et notamment d'évaluer l'impact possible d'un certain écart de la réalité à la théorie.

On va donc revenir sur les hypothèses essentielles, lesquelles ont été identifiées par des lettres au cours de l'exposé.

\section{Hypothèse A}

$u(t, x, y)$ étant le logarithme de la densité du poisson à l'endroit $(x, y)$ à l'instant $t$, selon l'hypothèse A: $Q\left(t, x_{1}, y_{1}, x_{2}, y_{2}\right)=E\left[u\left(t, x_{1}, y_{1}\right)-u\left(t, x_{2}, y_{2}\right)\right]^{2}$ ne dépend que de la distance $z_{12}$ des points $\left(x_{1}, y_{1}\right)$ et $\left(x_{2}, y_{2}\right)$.

a) $Q$ est supposé indépendant de $t$.

En fait il est possible d'estimer $Q$ en des époques différentes. C'est ce qui a été fait, les variations observées ne sont pas considérables à l'intérieur d'une saison de pêche dans l'exemple étudié. En revanche, les variations d'une année sur l'autre sont plus marquées. Ceci semble d'ailleurs da essentiellement à ce que la qualité des renseignements portés sur les carnets croit d'année en année. 
b) $Q$ est supposé indépendant de l'emplacement géographique.

C'est en fait l'homogénéité des fluctuations spatiales qui est postulée. Ce fut examiné sur notre exemple.

c) $Q$ ne dépend que de $z_{12}$ et non de $x_{1}-x_{2}$ et $y_{1}-y_{2}$ séparément.

Cette fois ce n'est plus l'homogénéité qui est supposée mais l'isotropie. Là encore sur l'exemple étudié ceci a été examiné. En fait $Q$ croît légèrement plus vite avec les écarts en latitude qu'en longitude. Il serait facile d'en tenir compte dans la programmation, mais la non isotropie évoquée est toutefois faible.

d) $Q$ est supposé ne pas varier avec la densité moyenne du poisson.

On pourrait concevoir que $Q$ ait tendance à être plus grand lorsque la densité moyenne croît. En fait il ne semble pas, une fois de plus sur l'exemple étudié qu'un tel phénomène soit significatif. Ce résultat est extrêmement important, il justifie en partie les méthodes choisies d'ajustement par les moindres carrés. Il serait nécessaire de choisir des pondérations plus sophistiquées dans le cas contraire.

Il est intéressant de noter que la méthode pourrait être modifiée si necessaire pour prendre en compte la dépendance de $Q$ vis-à-vis de tel ou tel facteur. Ne le faisant pas on opère en fait avec une valeur moyenne.

\section{Hypothèse B}

Selon cette hypothèse B la variance de $\log \left(N_{i k} / N_{j k}\right)$ est: $\operatorname{var}\left[\log \left(d_{i k} / d_{j k}\right)\right]+\operatorname{var}\left(\varepsilon_{i k}\right)+\operatorname{var}\left(\varepsilon_{j k}\right)$. Les variances s'ajoutent lorsqu'il n'y a pas d'interaction entre les différents termes d'une somme. L'interaction la plus plausible est celle liant les résidus aux densités: une haute densité pourrait provoquer une saturation de l'engin de pêche et donc des résidus négatifs dans les expressions du type $\log \left(N_{i k}\right)=\log \left(d_{i k}\right)+\log \left(P_{i}\right)+\varepsilon_{i k}$. Ceci est concevable à la limite dans l'exemple du germon de surface en début de saison. En fait d'ailleurs c'est le modèle de base $\log \left(N_{i k}\right)=\log \left(d_{i k}\right)+\log \left(\mathrm{P}_{i}\right)+\varepsilon_{i k}$ qui devient inadéquat.

\section{Hypothèse C}

L'hypothèse $\mathrm{C}$ conduit à supposer que la variance des résidus ne dépend pas du navire. Comme le montrera une note ultérieure consacrée à l'échantillonnage, il est possible d'obtenir une estimation grossière de la variance attachée à chaque navire. Pour la pêcherie de surface du germon il apparait ainsi que les canneurs possèdent une variance plus importante que les ligneurs, car ils sont plus inféodés au phénomènes de microstructures. Il serait possible de façon évidente de modifier la méthode de calcul des puissances locales en changeant simplement les pondérations.

\section{Hypothèse D}

En vertu de cette hypothèse $\log \left(N_{i k} / N_{j k}\right)$ et $\log \left(N_{i k+1} / N_{j k+1}\right)$ sont stochastiquement indépendants lorsqu'aux jours $k$ et $k+1$ les navires $i$ et $j$ ont opéré à une distance inférieure à une distance seuil $z c$. Elle suppose entre autres que $\log \left(d_{i k} / d_{j k}\right)$ et $\log \left(d_{i k+1} / d_{j k+1}\right)$ sont indépendants. Ceci est assuré par le choix d'une distance $z c$ assez faible: les structures de faible dimension sont moins stables dans le temps que les structures vastes, de plus les navires sont capables de se déplacer d'un jour sur l'autre renforçant encore cette indépendance.

Une autre cause de non indépendance pourrait venir de ce que les puissances varient au cours du temps, avec une certaine continuité. C'est alors en fait une puissance moyenne qui serait estimée, les écarts à cette moyenne étant intégrés dans les résidus. Si les écarts en question possèdent une certaine continuité, l'indépendance dans le temps des résidus n'est plus assurée. En fait, il est possible d'estimer la puissance sur une période restreinte et donc de suivre ainsi une possible évolution. Sur l'exemple étudié il est ainsi apparu que si la puissance ne variait pas de façon significative dans l'année, d'une année sur l'autre elle pouvait évoluer considérablement pour certains navires (modifications humaines ou matérielles).

De toutes façons, cette hypothèse d'indépendance peut être vérifiée. Pour cela la corrélation entre $\log \left(N_{i k} / N_{j k}\right)$ et $\log \left(N_{i k+1} / N_{j k+1}\right)$, les distances des deux navires étant restées inférieures à $z c$, est estimée. Pour la pêcherie de surface du germon cette estimation montre que l'indépendance des résultats des comparaisons faites deux jours consécutifs peut être raisonnablement admise lorsque $z c$ n'excède pas une soixantaine de miles. 
Pour conclure cette annexe B il faut noter que lorsque les hypothèses évoquées sont enfreintes les conséquences sont essentiellement de deux types: les pondérations ne sont plus optimales. Elles demeurent cependant raisonnables pour peu que l'écart aux hypothèses ne prenne des dimensions aberrantes; les inférences sont rendues fragiles, notamment lorsque l'hypothèse $D$ dite d'indépendance est loin d'être respectée.

En bref, les estimations obtenues restent raisonnables, et notamment pour l'essentiel non biaisées, les variances calculées devant être considérées comme indicatrice d'un ordre de grandeur, ou si l'on désire être prudent un majorant de la précision réelle des estimations obtenues.

\section{Annexe C \\ Estimation de $\operatorname{cov}\left(a l_{i 1}, a l_{i 2}\right)$; calcul de $\operatorname{cov}\left[\log \left(N_{i 1 k} / N_{j 1 k}\right), \log \left(N_{i 2 k} / N_{j 2 k}\right)\right]$}

Pour mener à bien ce calcul on remarquera d'abord que si l'on pose $p-s=(p-q)+(q-r)+(r-s)$ on obtient:

$\operatorname{var}(p-s)=\operatorname{var}(p-q)+\operatorname{var}(q-r)+\operatorname{var}(r-s)+2 \operatorname{cov}(p-q, r-s)+2 \operatorname{cov}(p-q, q-r)+2 \operatorname{cov}(q-r, r-s)$

Comme $\operatorname{var}(p-r)=\operatorname{var}(p-q)+\operatorname{var}(q-r)+2 \operatorname{cov}(p-q, q-r)$

et

$$
\operatorname{var}(q-s)=\operatorname{var}(q-r)+\operatorname{var}(r-s)+2 \operatorname{cov}(q-r, r-s)
$$

on parvient à la relation dite relation (1):

$$
2 \operatorname{cov}(p-q, r-s)=\operatorname{var}(p-s)+\operatorname{var}(q-r)-\operatorname{var}(p-r)-\operatorname{var}(r-s) .
$$

D'après le paragraphe II

$\operatorname{var}\left[\log \left(N_{i k} / N_{j k}\right)\right]=\operatorname{vari}\left(z_{i j k}\right)$ lorsque $i$ est différent de $j$, si $z_{i j k}$ est la distance des navires $i$ et $j$ le jour $k$. Lorsque $i=j$ bien évidemment $\operatorname{var}\left[\log \left(N_{i k} / N_{j k}\right)\right]$ est nulle.

On notera donc var $\left[\log \left(N_{i k} / N_{j k}\right)\right]=l_{i j} \operatorname{vari}\left(z_{i j k}\right)$.

Avec $\quad l_{i j}=1$ si $i \neq j, \quad l_{i j}=0$ si $i=j$

$2 \operatorname{cov}\left(\log \left(N_{i 1 k} / N_{j 1 k}\right), \log \left(N_{i 2 k} / N_{j 2 k}\right)\right)=l_{i 1 j 2} \operatorname{vari}\left(z_{i 1 j 2 k}\right)+l_{j 1 i 2} \operatorname{vari}\left(z_{j 1 i 2 k}\right)$

$-l_{i 112} \operatorname{vari}\left(z_{i 1 i 2 k}\right)-l_{j 1 j 2}$ vari $\left(z_{j 1 j 2 k}\right)$ d'après la relation (1).

Dans le cas où $i 1, i 2, j 1, j 2$ sont tous différents $\operatorname{cov}\left[\log \left(N_{i 1 k} / N_{j 1 k}\right), \log \left(N_{i 2 k} / N_{j 2 k}\right)\right]$ dépend des distances deux à deux des quatre navires $i 1, i 2, j 1, j 2$

$\begin{array}{llll}\frac{i 1}{\mathrm{x}} & \frac{j 1}{\mathrm{x}} & \frac{i 1}{\mathrm{x}} & \frac{j 1}{\mathrm{x}} \\ \frac{i 2}{\mathrm{x}} & \frac{j 2}{\mathrm{x}} & \frac{j 2}{\mathrm{x}} & \frac{i 2}{\mathrm{x}} \\ \text { configuration } 1 & & \text { configuration } 2\end{array}$

Si $i_{2}$ est proche de $i 1, j 2$ de $j 1$, si $i_{1}$ est éloigné de $j 2$ et $i_{2}$ de $j 1$

(configuration 1) $\operatorname{vari}\left(z_{i 1 j 2 k}\right)+\operatorname{vari}\left(z_{i 2 j 1 k}\right)>\operatorname{vari}\left(z_{i 1 i_{2 k}}\right)+\operatorname{vari}\left(z_{j 1 j 2 k}\right)$

$\operatorname{cov}\left[\log \left(N_{i 1 k} / N_{j 1 k}\right), \log \left(N_{i 2 k} / N_{j 2 k}\right)\right]$ est alors positif.

La configuration 2 est obtenue en échangeant $i 2$ et $j 2$. Ainsi $\operatorname{cov}\left[\log \left(N_{i 1 k} / N_{j 1 k}\right), \log \left(N_{i 2 k} / N_{j 2 k}\right)\right]$ change simplement de signe en gardant la même valeur absolue. Dans l'ensemble les configurations seront aléatoires et la valeur moyenne de $\operatorname{cov}\left[\log \left(N_{i 1 k} / N_{j 1 k}\right), \log \left(N_{i 2 k} / N_{j 2 k}\right)\right]$ sera nulle, une configuration ayant la même probabilité que celle obtenue en échangeant $i 2$ et $j 2$.

Comme on somme sur les différentes valeurs de $k$ on assimilera la moyenne à l'espérance et l'on négligera les termes où $i 1, i 2, j 1, j 2$ sont tous différents. La programmation en sera grandement simplifiée (quatre indices différents, conduisant à autant de boucles imbriquées aménent rapidement à des temps de calcul prohibitifs).

La covariance de $a l_{i 1}$ et $a l_{i 2}$ sera donc estimée par: 
Avec

$$
\begin{array}{ll}
1 / 2 \sum_{k, j 1 \neq i 1, j 2 \neq i 2} g(i 1, j 1, i 2, j 2, k) & {\left[l_{l 1 j 2} \operatorname{vari}\left(z_{i 1 j 2 k}\right)+l_{i 2 j 1} \operatorname{vari}\left(z_{i 2 j 1 k}\right)\right.} \\
& \left.-l_{i 1 i 2} \operatorname{vari}\left(z_{i 1 i 2 k}\right)-l_{j 1 j 2} \operatorname{vari}\left(z_{j 1 j 2 k}\right)\right]
\end{array}
$$

où

$$
\begin{aligned}
g(i 1, j 1, i 2, k) & =w_{i 1 j 1 k} w_{i 2 j 2 k} f(i 1, j 1, i 2, j 2) \\
f(i 1, j 1, i 2, j 2) & =0 \text { si } i_{1}, j 1, i 2, j 2 \text { sont tous différents } \\
& =1 \text { sinon. }
\end{aligned}
$$

Deux cas doivent alors être distingués:

a/ $i_{1} \neq i_{2}$

En développant les calculs on aboutit à estimer $\operatorname{cov}\left(a l_{i 1}, a l_{i 2}\right)$ par

$\mathrm{b} / i_{1}=i_{2}=i$

$$
\begin{aligned}
1 / 2 \sum_{k_{, j} j} & \left\{w_{i 1 j k} l_{i 1 j} w_{i 2 j k} l_{i 2 j}\left[\operatorname{vari}\left(z_{i 1 j k}\right)+\operatorname{vari}\left(z_{i 2 j k}\right)\right]\right\} \\
- & {\left[\operatorname{vari}\left(z_{i 1 i 2 k}\right) w_{i 1 i 2 k}\left(w_{i 2 j k} l_{i 2 j}+w_{i 1 j k} l_{i 1 j}\right)\right] }
\end{aligned}
$$

La variance de $a l_{i}$ est estimée par $\sum_{k, j} w^{2} i j k$ vari $\left(z_{i j k}\right)$.

\section{Annexe D}

\section{Remarque sur la non optimalité des estimateurs des puissances locales}

Il a été noté que l'estimateur des puissances de pêche locales développé au paragraphe III n'était pas l'estimateur du maximum de vraisemblance. Celui-ci est en effet calculable en théorie, mais la programmation des formules obtenues deviendrait impossible. Il n'est cependant pas exclu de trouver un estimateur meilleur que celui proposé, dont l'inconvénient essentiel réside en ce que si un jour un bateau donné a été comparable à de nombreux autres, le résultat de la pêche de ce bateau en ce jour aura une importance peut être excessive sur l'estimation de sa puissance de pêche. En effet, en ce jour de nombreuses comparaisons du type $\log \left(N_{i k} / N_{j k}\right)$ auront été opérées, et la méthode suggérée est en fait un ajustement des comparaisons théoriques $\log \left(P_{i} / P_{j}\right)$ aux comparaisons observées $\log \left(N_{i k} / N_{j k}\right)$. 